\title{
On the dynamics of rocking motion of single rigid-block structures
}

\author{
F. Peña ${ }^{1, *, \dagger}$, F. Prieto ${ }^{1}$, P. B. Lourenço ${ }^{1}$, A. Campos Costa $^{2}$ and J. V. Lemos ${ }^{2}$ \\ ${ }^{1}$ Department of Civil Engineering, University of Minho, Azurém, 4800-058 Guimarães, Portugal \\ ${ }^{2}$ Laboratório Nacional de Engenharia Civil, Av Brasil 101, 1700-066 Lisboa, Portugal
}

\begin{abstract}
SUMMARY
This paper describes the behavior of single rigid-block structures under dynamic loading. A comprehensive experimental investigation has been carried out to study the rocking response of four blue granite stones with different geometrical characteristics under free vibration, and harmonic and random motions of the base. In total, 275 tests on a shaking table were carried out in order to address the issues of repeatability of the results and stability of the rocking motion response. Two different tools for the numerical simulations of the rocking motion of rigid blocks are considered. The first tool is analytical and overcomes the usual limitations of the traditional piecewise equations of motion through a Lagrangian formalism. The second tool is based on the discrete element method (DEM), especially effective for the numerical modeling of rigid blocks. A new methodology is proposed for finding the parameters of the DEM by using the parameters of the classical theory. An extensive comparison between numerical and experimental data has been carried out to validate and define the limitations of the analytical tools under study. Copyright (C) 2007 John Wiley \& Sons, Ltd.
\end{abstract}

Received 1 February 2006; Revised 20 June 2007; Accepted 21 June 2007

KEY WORDS: rigid blocks; rocking motion; dynamics; discrete element method; complex coupled rocking rotations method

\section{INTRODUCTION}

The problem of a rigid body $(\mathrm{RB})$ on a rigid foundation experiencing lateral dynamic loading has been of interest to researchers for many years [1-9]. A number of analytical and numerical models have been proposed in order to study this phenomenon.

\footnotetext{
${ }^{*}$ Correspondence to: F. Peña, Instituto de Ingeniería, Edificio 2-315, Circuito Escolar, Ciudad Universitaria, 04510 Mexico City, Mexico.

${ }^{\dagger}$ E-mail: fpem@pumas.iingen.unam.mx

Contract/grant sponsor: Fundação para a Ciência e a Tecnologia; contract/grant numbers: SFRH/BPD/17449/2004, SFRH/BD/9014/2002

Contract/grant sponsor: Ecoleader Group 4
}

Copyright (C) 2007 John Wiley \& Sons, Ltd. 
The reference analytical frame for the study of RM dynamics remains the formulation introduced by Housner [4] (which will be referred as classical theory in the present study). This method tackles the dynamical problem with two piecewise equations of motion for each sign of the rocking angle, while damping is reproduced by means of a coefficient of restitution. Nevertheless, Housner's classical formulation presents the following drawbacks: (a) the theory makes the application of standard mathematical theorems and techniques from the theory of differential equations very difficult; and (b) its generalization to a higher number of blocks becomes intractable when the number of degrees of freedom increases.

An alternative formulation [10], known as the complex coupled rocking rotations (CCRR) method, in which the rocking angle $\theta$ is assumed to be a complex quantity, was shown to be a useful tool in the study of RM. The aim of the CCRR method is to unify both piecewise equations and impact mechanisms into a model in which the motion is governed by a single differential equation. This approach allows to study configurations involving a higher number of blocks taking advantage of the scalar nature of a fully Lagrangian-Hamiltonian formulation. Damping effects are understood by means of a generalized Dirac- $\delta$ force [11], which is in agreement with the experimental results.

On the other hand, several authors [12-17] have used the discrete element method (DEM) to study the rocking motion of RB. Those works show that DEM is a useful tool in the study of RB in free rocking motion or subjected to a base motion. However, in the referred contributions, the parameters that define the characteristics of the model (mainly stiffness and damping) are found by using fitting numerical results to experimental tests, which is a major limitation of this method for engineering applications. For this reason, a methodology for finding the parameters of DE model based on the parameters of the classical formulation is proposed here.

An extensive experimental investigation has been carried out to study the rocking response of four blue granite stones with different geometrical characteristics under free vibration, harmonic and random motions of the base. A set of 275 tests were reproduced with CCRR and DE models in order to show the response and limitations of the analytical tools in the RM problem. Therefore, the present paper also describes the numerical modeling of the experimental tests carried out on a shaking table.

\section{NUMERICAL MODELS}

In this section, a brief review of the formulation of the adopted numerical models is presented. The classical formulation [4] is also described in order to understand better the differences and similarity between models, even if this formulation is not used here. The complete CCRR formulation can be found in [10], while the description of DEM was taken from [18, 19]. A MatLab code, developed by the authors according to [10], and the commercial UDEC code [18], based on the DEM, have been used to perform the numerical analyses.

\subsection{Classical formulation}

It has been observed in experimental tests that there are three main parameters for the planar rocking motion problem. These quantities depend solely on the geometry of the specimens, as follows: $\alpha$ is defined as the angle at which the stone overturns due to static forces, it is called critical angle; $p$ is the frequency associated with the system due to the interaction between the 

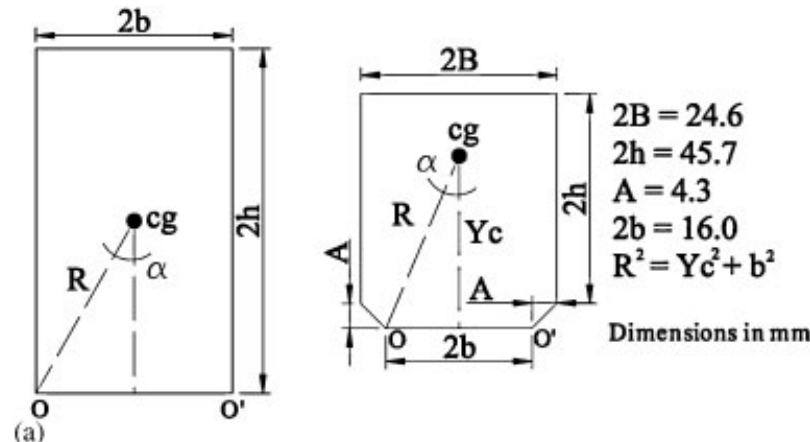

Figure 1. Test specimens: (a) geometry of the blocks tested. Stones 1, 2, 3 (left) and 4 (right) and (b) single blocks.

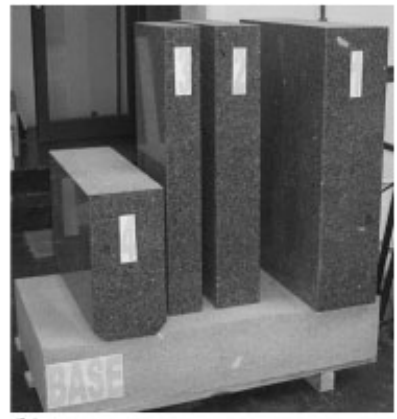

(b)

block and its base and $\mu$ is the so-called coefficient of restitution and it is defined as the angular velocity reduction ratio between two consecutive impacts.

These three parameters will be referenced in this work as theoretical parameters since they govern the differential equation of RM of a RB. For a rectangular block, with a base $2 b$ and a height $2 h$ (Figure 1(a)), subjected to horizontal ground acceleration $a(\tau)$, the differential equations are derived by considering the equilibrium of moments about the centers of rotation $O-O^{\prime}$ at the block base

$$
\theta^{\prime \prime} \pm p^{2} \sin (\alpha \mp \theta)=p^{2} \cos (\alpha \mp \theta) \frac{a(\tau)}{g}
$$

where $\left({ }^{\prime}\right)$ means differentiation with respect to time $\tau, g$ is the acceleration of gravity, the \pm sign refers to the domains of the rocking angle $\theta>0$ and $\theta<0$, respectively, while $\alpha$ is equal to

$$
\alpha=\tan ^{-1}\left(\frac{b}{h}\right)
$$

and $p$ is a parameter with dimensions of frequency defined as

$$
p^{2}=\frac{M g R}{I}
$$

Here, $M$ is the mass, $I=\left(\frac{4}{3}\right) M R^{2}$ is the corresponding moment of inertia (defined with respect to $O$ or $O^{\prime}$ ) and $R$ is defined as $R=\sqrt{b^{2}+h^{2}}$. For rectangular blocks $p^{2}$ reduces to $3 g / 4 R$.

In order to take into account the impact mechanisms, Housner [4] assumed a coefficient of restitution $\mu$ that multiplies the angular velocity $\theta^{\prime}$ when the block passes through the equilibrium position at $\theta=0$. By considering that angular moment conservation exists before and after an impact, the coefficient of restitution can be obtained by means of

$$
\mu=\frac{\theta^{\prime \mathrm{a}}}{\theta^{\prime \mathrm{b}}}=1-\frac{3}{2} \sin ^{2}(\alpha)
$$

where $\theta^{\prime a}$ and $\theta^{\prime b}$ are the angular velocities just after and before the impact, respectively. 


\subsection{Complex coupled rocking rotations method}

In free rocking motion a symmetry in the system is present. The symmetry is equivalent to an invariance with respect to the sign of rocking angle $\theta$, and as a consequence, an associated conserved magnitude exists. This fact can be exploited by expressing $\theta$ as complex quantity, with a module $r$ and a phase $\psi$, by means of

$$
\theta=r \mathrm{e}^{\mathrm{i} \psi}
$$

Here, the sign of $\theta$ is only associated with $\psi$ and $r$ equals the absolute value of $\theta$. Therefore, the Lagrangian function is built as

$$
L_{0}=\frac{I}{2}\left(r^{\prime 2}+r^{2} \psi^{\prime 2}\right)-M g R \cos (\alpha-r)
$$

The impact forces and the base motion acceleration are taken into account in the D'Alembert equation as generalized forces:

$$
\begin{aligned}
& \frac{\partial}{\partial \tau}\left(\frac{\partial L}{\partial r^{\prime}}\right)-\frac{\partial L}{\partial r}=Q_{r}^{\mathrm{d}}+Q_{r}^{\mathrm{s}} \\
& \frac{\partial}{\partial \tau}\left(\frac{\partial L}{\partial \psi^{\prime}}\right)-\frac{\partial L}{\partial \psi}=Q_{\psi}^{\mathrm{d}}+Q_{\psi}^{\mathrm{s}}
\end{aligned}
$$

Here, $Q_{r}^{\mathrm{d}}$ and $Q_{r}^{\mathrm{s}}$ represent the generalized damping force and base excitation with respect to the canonical variable $r$, respectively. The same holds for $Q_{\psi}^{\mathrm{d}}$ and $Q_{\psi}^{\mathrm{s}}$ for $\psi$. These variables are defined as [10]

$$
\begin{aligned}
Q_{r}^{\mathrm{d}} & =\left(\frac{\ln (\mu) I}{\pi}\right) \psi^{\prime} r^{\prime} \\
Q_{r}^{\mathrm{s}} & =M a(\tau) R \cos (\alpha-r) \cos (\psi) \\
Q_{\psi}^{\mathrm{d}} & =0 \\
Q_{\psi}^{\mathrm{s}} & =0
\end{aligned}
$$

with $a(\tau)$ being the base acceleration. By normalizing the module $\chi=r / \alpha$ and considering the adimensional time $t=p \tau$, the next differential equation is obtained [10]:

$$
\ddot{\chi}=\frac{l^{2}}{\chi^{3}}-\frac{1}{\alpha} \sin (\alpha-\alpha \chi)+\frac{2 \ln (\mu)}{\pi \chi^{2}} \dot{\chi} l+\frac{a(t / p)}{g \alpha} \cos (\alpha-\alpha \chi) \cos (\psi)
$$

where ( ) means derivation with respect to adimensional time $t$. Equation (10) is numerically integrated by means of Runge-Kutt-Fehlberg method by using a constant time step $\Delta t$ and giving the initial conditions, as well as the classical parameters $(\alpha, p$ and $\mu)$.

\subsection{Discrete element method}

DEM can be considered as a method for modeling discontinuous media. This analysis technique allows relative motion between elements, which is especially suitable for problems in which the 
relative motion between blocks is a significant part of the deformation. It is possible to represent the material as rigid or deformable. The first option is used here because in the rocking motion, most of the displacements are due to the relative motion between elements and not due to the deformation of the materials. However, the contact between elements (joint) has not been considered as completely rigid because the energy dissipation at each impact depends on the material of the block and its base [20].

The contact between elements is defined as sets of contact points located at the corner of the elements. The contact point is defined by two linear springs, one axial and one shear and a viscous damper. The axial spring is linear-elastic in compression, while zero tensile strength is assumed for tension. The shear spring is defined as linear-elastic-perfectly plastic and a Coulomb-type behavior is assumed. Therefore, four parameters are needed to define the mechanical behavior of the contact: axial stiffness $K$, shear stiffness $K_{\mathrm{s}}$, friction angle $\phi$ and cohesion $c$. The viscous damping $C$ is regarded as a mass $M$ and stiffness $K$-dependent quantity by means of the Rayleigh formulation $C=a M+b K$, where $a$ is the mass proportional damping constant and $b$ is the stiffness proportional damping constant.

\section{EXPERIMENTAL TESTS}

The numerical models proposed here have been verified by comparing their responses with the results obtained from extensive experimental tests on the rocking response of $\mathrm{RB}$. The tests were performed at the shaking table of the National Laboratory of Civil Engineering (LNEC) of Portugal on four single RBs (blue granite stones), subjected to different types of base motion: (a) free rocking motion, (b) harmonic motion and (c) random motion.

\subsection{Characteristics of the specimens}

The experimental tests were carried out on four single blue granite stones specifically manufactured for the tests (Figure 1(b)). Each stone had different geometrical dimensions (Table I). The dimensions of the specimens 1,2 and 3 were fixed to achieve a height-width ratio $(h / b)$ of 4 , 6 and 8 , respectively. The height was equal to $1 \mathrm{~m}$ which was kept constant for the three specimens. In order to minimize the three-dimensional (3D) effects, the blocks were designed with a thickness-width ratio $(t / b)$ of 3 . With the aim of reducing the continuous degradation at the block corners occurring at every impact, the stones were manufactured with a small cut oriented at $45^{\circ}$ at their bases. The size of the cut was $5 \mathrm{~mm}$.

Table I. Test specimen characteristics.

\begin{tabular}{lccccc}
\hline Specimen & $\begin{array}{c}\text { Width } \\
2 b(\mathrm{~m})\end{array}$ & $\begin{array}{c}\text { Height } \\
2 h(\mathrm{~m})\end{array}$ & $\begin{array}{c}\text { Thickness } \\
2 t(\mathrm{~m})\end{array}$ & $\begin{array}{c}\text { Mass } \\
(\mathrm{kg})\end{array}$ & $\begin{array}{c}\text { Inertia } \\
I_{0}\left(\mathrm{~kg} \mathrm{~m}^{2}\right)\end{array}$ \\
\hline 1 & 0.25 & 1.000 & 0.754 & 503 & 179 \\
2 & 0.17 & 1.000 & 0.502 & 228 & 78 \\
3 & 0.12 & 1.000 & 0.375 & 120 & 42 \\
4 & 0.16 & 0.457 & 0.750 & 245 & 26 \\
Base & 1.00 & 0.250 & 0.750 & 500 & - \\
\hline
\end{tabular}



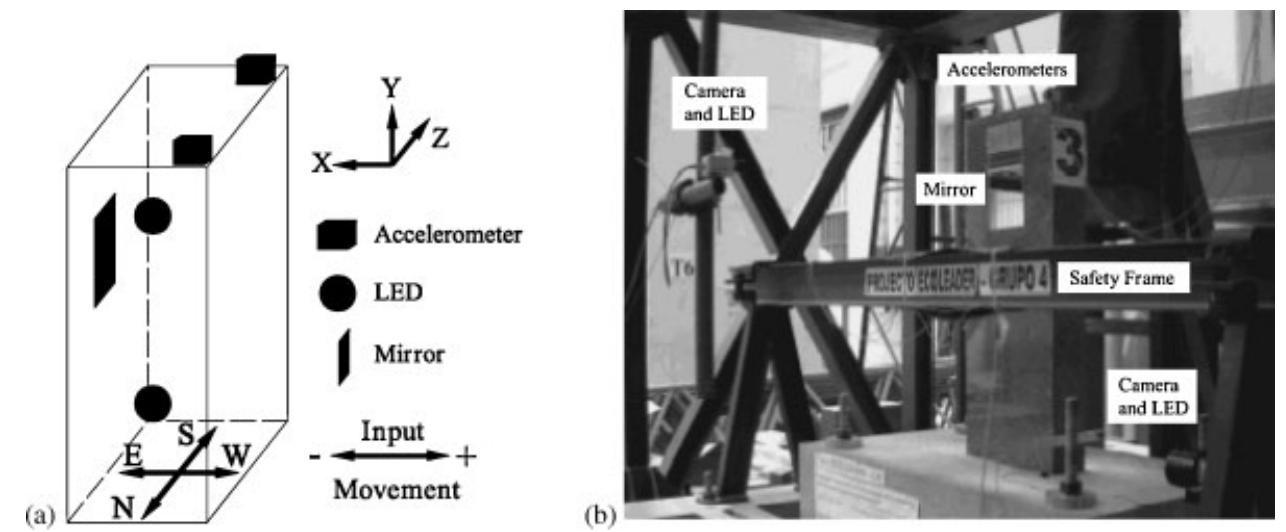

Figure 2. Test setup: (a) reference system of the data acquisition system and typical location of LEDs in the RB and (b) view of the test setup.

In addition, specimen number 4 was specifically designed with a different geometry in order to compare its performance with the rest of the stones. It had a large $45^{\circ}$ cut $(40 \mathrm{~mm})$ at the base (Figure 1). Moreover, a foundation of the same material was used as the base where the blocks were free to rock. This foundation was fixed to the shaking table by means of four steel bolts.

\subsection{Test set up}

The data acquisition system was designed to describe the position of the specimens at each instant of the test and, simultaneously, to avoid the possibility of the system influencing the response of the specimens. In this context, the data acquisition was based upon monitoring light emission diode systems (LEDs) by means of high-resolution cameras. This eliminates noise errors and enables accurate position measurement. The position resolution obtained was of $\frac{1}{5000}$.

The main data obtained were rotations around the $Y$ - and $Z$-axes, and linear displacements $X$ and $Y$. The system coordinate is depicted in Figure 2(a). Rotations $Y$ and $Z$ were directly measured by means of a mirror linked to the block's surface on the West face of the specimens. The LED was located at the same position of the measuring camera and the light ray emitted is reflected at the mirror, so that a large measured range is obtained $( \pm 100 \mathrm{~mm})$. Two accelerometers were placed at the top of each block. One triaxial accelerometer was located in the north face and one biaxial accelerometer was located in the south face. The displacements and accelerations of the shaking table were also measured (Figure 2(b)).

\subsection{Types of base motion}

In order to study the dynamic behavior of the RB, three different tests were carried out: (a) free rocking motion, (b) harmonic motion and (c) random motion. The first type of test allows to identify the parameters used in the classical theory and at the same time to calibrate the analytical models. On the one hand, the harmonic tests allow to study, in a simple way, the dynamic behavior of single blocks undergoing RM regime, while on the other hand, the behavior of the RB under earthquake conditions was studied with the random test. 
Thirty synthetic earthquake compatibles with the design spectrum proposed by the Eurocode 8 [21] were generated. In order to identify them, they were named consecutively with the number of generation. The constant branch of the spectrum was located between 0.1 and $0.3 \mathrm{~s}$, with a spectral acceleration of $7 \mathrm{~m} / \mathrm{s}^{2}$ while the maximum ground acceleration was $2.8 \mathrm{~m} / \mathrm{s}^{2}$. The main aim of the study is to address the stability of RM under random motion.

\section{SOME CONSIDERATIONS FOR THE ROCKING MOTION ANALYSIS}

The analytical models are very sensitive to parameters such as block parameters (stiffness and/or geometrical parameters), damping (impact), etc. $[10,14,17]$. Thus, preliminary analyses were carried out in order to fully understand the limitations and sensitivity of the analytical models with respect to variations in the values of these parameters.

\subsection{Free rocking motion}

The theoretical parameters give useful information about the characteristics of the RM. They can be obtained by Equations (2)-(4) or by means of free rocking motion tests. It is well known that the experimental values are not equal to the theoretical values, because the hypotheses assumed by the classical theory are not complete fulfilled.

The experimental parameters were adjusted by means of a minimized error surface [8]. Since for free RM, the energy is conserved between two consecutive impacts, it is possible to derive an analytical expression for the time $(\tau)$ of the maximum amplitudes of rocking angle $(\theta)$, before and after impact $n$, given by

$$
\tau_{n}=\frac{1}{p \sqrt{2}} \int_{r_{n-1}}^{0} \frac{\mathrm{d} x}{\sqrt{\cos (x-\alpha)-\cos (\alpha)}}
$$

On the other hand, taking into account that the relation between energy after the $n$th impact $E_{n}$ and the initial energy $E_{0}$ is given by $E_{n}=E_{0} \mu^{2 n}$, a useful formula for the coefficient of restitution $\mu$ can be derived as

$$
\mu_{n}=\left(\frac{\cos \left(\alpha-r_{n}\right)-\cos (\alpha)}{\cos \left(\alpha-r_{0}\right)-\cos (\alpha)}\right)^{1 / 2 n}
$$

where $r_{n}$ is the rocking angle amplitude after the $n$th impact. Equation (11) allows us to find the experimental values for $\tau_{n}^{*}$ and $r_{n}^{*}$. The parameters have been fitted following the next procedure.

- Parameters $\alpha$ and $p$ are obtained by minimizing the error surface:

$$
\Sigma(\alpha, p)=\Sigma_{n}\left(\tau_{n}-\tau_{n}^{*}\right)^{2}
$$

- Parameter $\mu_{n}$ is derived using Equation (12), using the fitted value of $\alpha$ and the experimental amplitudes $r^{*}$.

- The final value of $\mu$ is obtained by averaging the recorded values of $\mu_{n}$ in the different impacts.

Table II shows the theoretical and experimental values of these parameters. Experimental parameters for specimens 1 and 2 are similar to the theoretical values, with differences smaller 
Table II. Theoretical and experimental classic parameters.

\begin{tabular}{|c|c|c|c|c|c|c|c|c|c|}
\hline \multirow[b]{2}{*}{ Specimen } & \multicolumn{3}{|c|}{$\alpha(\mathrm{rad})$} & \multicolumn{3}{|c|}{$\mu$} & \multicolumn{3}{|c|}{$p\left(\mathrm{~s}^{-1}\right)$} \\
\hline & $T$ & $E$ & $\%$ & $T$ & $E$ & $\%$ & $T$ & $E$ & $\%$ \\
\hline 1 & 0.242 & 0.235 & -2.9 & 0.914 & 0.936 & 2.4 & 3.78 & 3.84 & 1.6 \\
\hline 2 & 0.168 & 0.163 & -3.0 & 0.958 & 0.973 & 1.6 & 3.81 & 4.05 & 6.3 \\
\hline 3 & 0.119 & 0.154 & 29.4 & 0.978 & 0.978 & 0.0 & 3.82 & 3.61 & -5.5 \\
\hline 4 & 0.310 & 0.268 & -13.5 & 0.860 & 0.927 & 7.8 & 5.16 & 5.02 & -2.7 \\
\hline
\end{tabular}

Note: $T$, theoretical; $E$, experimental; $\%$, error (in percentage).

than 3\%. On the other hand, specimens 3 and 4 present significant differences in their parameters, especially for $\alpha$, with errors around 30 and $13 \%$, respectively.

The small changes for specimens 1 and 2 are related to the small cuts at $45^{\circ}$ at the base of the specimens. In fact, if we keep the height constant and vary the base in order to obtain the same $\alpha$, the bases for specimens 1 and 2 must be reduced to $10 \mathrm{~mm}$.

For specimen 4 , the complexity of the adopted shape can probably justify the difference observed. Since $\alpha$ is the angle formed by the distance from the base to the gravity center and the distance from the middle point of the base to the 'rotation point'. On the one hand, when the block is completely rigid, the rotation point is located at the corner of the base. On the other hand, when the block is not completely rigid, the rotation point is not located at the corner of the base. In fact, in this case there does not exist a rotation point. The block will tend to rotate not on a single point but on a finite surface. The size of this surface will depend on the material stiffness, it will tend to zero (single point) when the stiffness of the material tends to infinity.

Finally, the increment of $30 \mathrm{~mm}$ in the base of specimen 3 obtained can be explained by the $3 \mathrm{D}$ effects recorded in this block (Figure 3(a)). Specimen 3 shows a vibration around vertical axis (torsion). This vibration around the $Y$-axis means that stone 3 behaves like a stone with a larger base as shown schematically in Figure 3(b). This effect related to the high slenderness of the specimen $(h / b$ equal to 8$)$ causes the theoretical parameters to be those of a block with an equivalent larger base.

The coefficient of restitution, $\mu$, is related to damping of the system. The experimental values are slightly higher than the theoretical values because there is no conservation of angular moment, since the bodies are not fully rigid. On the other hand, $p$ can be defined as the frequency of the system due to the interaction between the block and its base, and it presents a random variation with respect to the theoretical parameters. These differences are due to the microscopical roughnesses of the contact surfaces.

The experimental tests have shown that the experimental values do not match with the theoretical due to (a) detailed measurements in the real dimensions of the blocks; (b) rotation surface; and (c) $3 \mathrm{D}$ effects. Therefore, the minimization process proposed in this paper overcomes the difficulties for extracting the value of the theoretical parameters.

\subsection{Derivation of DE model parameters}

The parameters required in the CCRR model are the same as the classical formulation. It means that the $\alpha, p$ and $\mu$ parameters are sufficient for defining the properties of the model. On the other hand, the following additional parameters are required in the DE model definition: axial $K$ and 

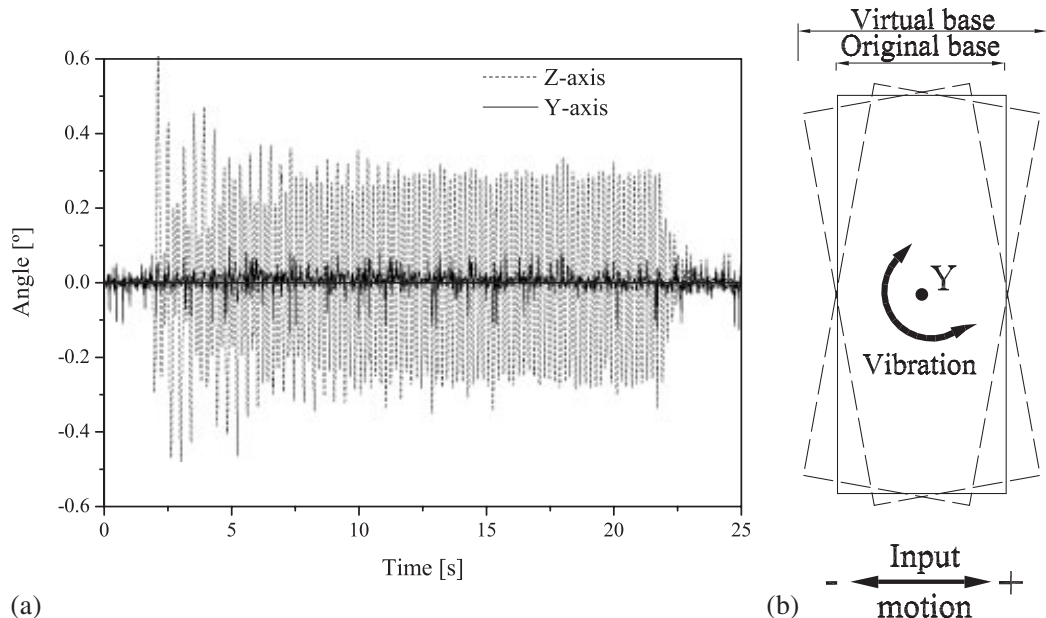

Figure 3. Specimen 3: (a) rocking and total torsion angle (constant sine $5 \mathrm{~Hz}$ and $2 \mathrm{~mm}$ ) and (b) schematic vibration around $Y$-axis (plan view).

shear $K_{\mathrm{s}}$ stiffness, cohesion $c$ and friction angle $\phi$, as well as the damping parameters $\xi_{\min }$ and $f_{\min }$.

The parameter $p$ can be defined as the frequency associated with the system due to the interaction of the block with its base. In this context, it is possible to define the axial stiffness $K$ as an average value:

$$
K=M p^{2}=K_{1}
$$

By taking into account the theoretical value of $p$ (Equation (3)), $K$ takes the following value:

$$
K=M \frac{W R}{I}=K_{2}
$$

It is clear that $K_{1}$ will be equal to $K_{2}$ if theoretical parameters are used; however, if the fitting parameters are used then $K_{1}$ will be different from $K_{2}$. For this reason, $K$ is defined as an average value

$$
K=\frac{K_{1}+K_{2}}{2}=\frac{M}{2}\left(p^{2}+\frac{W R}{I}\right)
$$

and for a rectangular block, Equation (16) becomes:

$$
K=\frac{M}{2}\left(p^{2}+\frac{3 g}{4 R}\right)
$$

Shear stiffness $K_{\mathrm{S}}$ is considered equal to axial stiffness $K[14,16,17]$, while the cohesion $c$ is considered null, as typical of dry joints. The $\alpha$ parameter can be defined as the relationship between the rotation points of the base $\left(O-O^{\prime}\right)$ and the position of the gravity center $(\mathrm{cg})$ of the block. In theory, these rotation points are located at the corners of the block; however, this is true only if the block and its base are completely rigid. In practice, the rotation points are not located at 
the corners of the block, because the material is not perfectly rigid. In the DE model, the rotation points are located at the corners of the blocks. Thus, it is necessary to consider an equivalent base $\left(b_{\text {eq }}\right)$, in order to take into account the real value of the $\alpha$ parameter, defined as

$$
b_{\text {eq }}=h \tan (\alpha)
$$

\subsection{Calibration of numerical models-theoretical and fitting parameters}

The free rocking motion tests were used for calibrating the CCRR and the DE models. The first step in the calibration is to calculate the response of both models using the values of the theoretical parameters (Equations (2)-(4); Table II). Table III shows the values used for defining the DE model. The friction angle, $\phi$, is considered to be equal to $30^{\circ}$ [22] for the four specimens. It is worth noting that the stiffness was calculated using Equation (17), while the damping $\xi_{\min }$ was fitted in order to obtain the best correlation with the experimental test data. The frequency $f_{\min }$ was considered equal to $p$ (see next subsection).

Figure 4(a) shows the comparison on a typical free rocking test with the response obtained from both models. It can be seen that the response obtained from the numerical models do not fully agree with the experimental test data. In particular, the differences between models and experiments lie inside the period and amplitude range of each cycle.

Table III. Parameters used in the definition of the DE models.

\begin{tabular}{|c|c|c|c|c|c|c|c|c|}
\hline \multirow[b]{2}{*}{ Specimen } & \multicolumn{2}{|c|}{$K(\mathrm{~N} / \mathrm{m})$} & \multicolumn{2}{|c|}{$\xi\left(\times 10^{-3}\right)$} & \multicolumn{2}{|c|}{$f_{\min }(\mathrm{Hz})$} & \multicolumn{2}{|c|}{$2 b_{\mathrm{eq}}(\mathrm{m})$} \\
\hline & $T$ & $F$ & $T$ & $F$ & $T$ & $F$ & $T$ & $F$ \\
\hline 1 & 7233 & 6560 & 2.92 & 4.33 & 3.78 & 3.84 & 0.25 & 0.24 \\
\hline 2 & 3302 & 3449 & 1.75 & 2.34 & 3.81 & 4.05 & 0.17 & 0.16 \\
\hline 3 & 1174 & 2168 & 5.00 & 0.73 & 3.82 & 3.61 & 0.12 & 0.15 \\
\hline 4 & 6454 & 6471 & 1.50 & 3.09 & 5.16 & 5.02 & 0.16 & 0.14 \\
\hline
\end{tabular}

Note: $T$, theoretical; $F$, fitting.
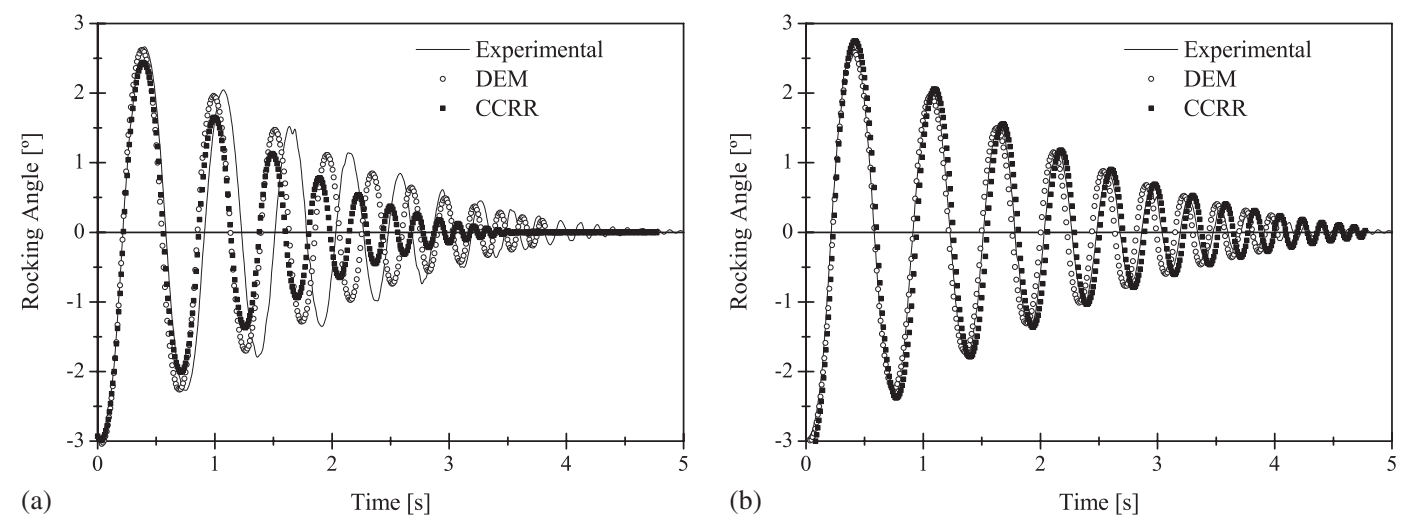

Figure 4. Typical free rocking motion response of DE and CCRR models: (a) theoretical parameters and (b) fitting parameters. 
The numerical models are very sensitive to variations in classical parameters particularly parameter $\alpha$. The fitting parameters obtained according to the minimization procedure (Table II) have variations smaller than 5\%. However, these small variations induce large differences in the response of the numerical models. Good agreement has been achieved between numerical and experimental results (Figure 4(b)) when fitted parameters are used (Tables II and III), even in the last cycles of RM. It should be highlighted that the damping of the DE model was fitted (Table III) in order to calibrate the model. With these values, an empirical formula was proposed for the calculation of the equivalent damping which is explained in the next subsection.

\subsection{Impact and equivalent viscous damping in the DE model}

The damping force appears in the system only during the impact as a Dirac- $\delta$ force. As the damping in the DE model is considered viscous and the Rayleigh formulation is used, some considerations must be made in order to take into account the damping in the real system due to the impact. The damping in the DE model is related to each contact point. Thus, when the contact point is detached from the base, the damping must be zero. Therefore, both terms damping proportional to the mass and stiffness must be zero. On the one hand, the damping proportional to the mass will be zero only if $a=0$. On the other hand, the proportional stiffness damping factor $b$ will be responsible for introducing the impulsive damping force. This is possible because the stiffness is zero for tensile strength, whereas the stiffness will reach a finite value when contact takes place.

With the damping factors obtained using the calibration of the DE model with inverse fitting (Table III), it is possible to obtain an empirical formula for the equivalent viscous damping. This formula is a function of the coefficient of restitution, $\mu$, and the generalized damping factor, $\Gamma$, that relates the generalized damping force $Q^{\mathrm{d}}$ with the viscous damping $C$. As $C$ depends only on the stiffness $K$ and $Q^{\mathrm{d}}$ depends on the geometry of the block (Equation (9)), $\Gamma$ can be defined as

$$
\Gamma=K R=\frac{M}{2}\left(R p^{2}+\frac{3}{4} g\right)
$$

In Figure 5, the values of factor $b$ versus the ratio $\Gamma / \mu^{2}$ for the four specimens are plotted. The best curve fitting is logarithmic and can be calculated as

$$
\sqrt{b}=0.0057 \ln \left(\frac{\Gamma}{\mu^{2}}\right)-0.0336
$$

As $p$ can be considered as the associated frequency of the system due to the interaction of the block and its base, the frequency $f_{\min }$ associated with the damping $\xi_{\min }$ can be calculated as

$$
f_{\min }=p
$$

Finally, the damping $\xi_{\min }$ is obtained by means of

$$
\xi_{\min }=2 \pi b f_{\min }
$$




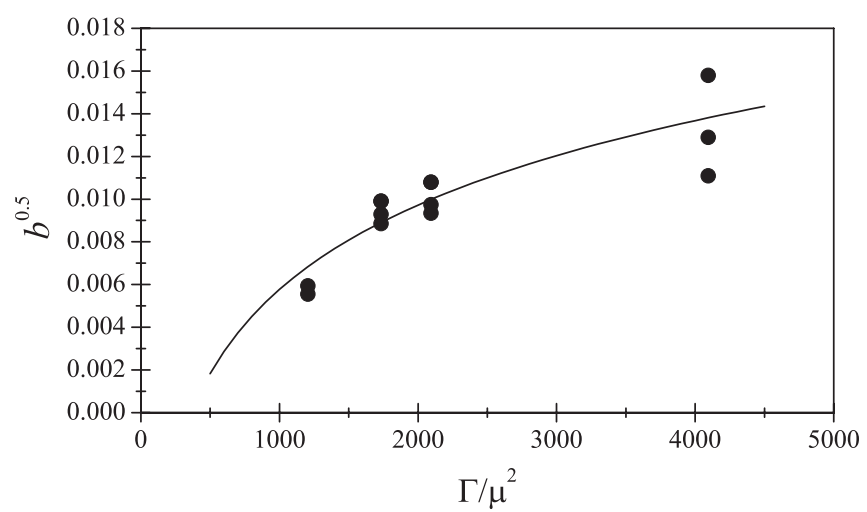

Figure 5. Curve fitting for empirical formulation of equivalent damping.

\section{NUMERICAL SIMULATIONS}

The numerical simulations were carried out with the CCRR and DE models. These codes use the parameter values obtained from free rocking tests defined in Section 4. Their values remain unchanged for all harmonic and random simulations. The 275 tests were reproduced with both the models in order to test the performance of the analytical tools in the rocking motion problem. In order to take into account some possible influence of the shaking table in the response of the blocks, numerical analyses were performed using the recorded motion of the table as the base excitation for the blocks.

\subsection{Harmonic motion}

The response of a RB under harmonic motion depends on the frequency and the amplitude of the harmonic motion. To illustrate this fact, Table IV shows typical frequencies and amplitudes at which a specimen shows rocking. A clear relationship between the harmonic motion and the rocking motion exists. It is clear that if the amplitude increases while the frequency remains constant, the amplitude of the rocking increases too. On the other hand, if the frequency of the motion increases, while the amplitude of the load remains constant, the rocking angle decreases. Thus, there are couples of frequencies and amplitudes of harmonic motion leading the RB to experience rocking. The set of these couples is called rocking motion space.

Some tests were repeated in order to study the repeatability of the response of the RB under forced excitation. The tests selected for repetition were: (a) specimen 1 with the Hanning sine with frequency of $3.3 \mathrm{~Hz}$ and amplitude of $7 \mathrm{~mm}$; (b) specimen 2 with constant sine with frequency of $3.3 \mathrm{~Hz}$ and amplitude of $6 \mathrm{~mm}$; (c) specimens 3 and 4 with all the constant sine tests. All these tests show that the repeatability in the harmonic motion exists.

The six tests performed with specimen 1 subjected to Hanning sine of $3.3 \mathrm{~Hz}$ and amplitude of $7 \mathrm{~mm}$ are depicted in Figure 6(a). The response of all six tests is similar. Taking the first test as reference, the differences (in percentage) in the values of the maximum rocking angle are found to be below 3\%, except for test number 5 that shows an error of $10 \%$. Figure 6(b) shows the Fourier spectra of the rocking angle. It can be seen that all six spectra are practically the same and they show little differences in frequencies up to $10 \mathrm{~Hz}$. The Fourier spectra show two peaks. 
Table IV. Typical rocking motion space and maximum rocking angle (deg.) for harmonic motion (specimen 2).

\begin{tabular}{lccccccc}
\hline & \multicolumn{7}{c}{ Amplitude (mm) } \\
\cline { 2 - 8 } Constant sine $(\mathrm{Hz})$ & 03 & 04 & 05 & 06 & 08 & 10 & 12 \\
\hline 0.5 & $\mathrm{~N}-\mathrm{R}$ & & & & & & N-R \\
1.0 & & & & & & & N-R \\
1.5 & & $\mathrm{~N}-\mathrm{R}$ & $\mathrm{N}-\mathrm{R}$ & $\mathrm{N}-\mathrm{R}$ & 3.04 & 3.93 & \\
2.0 & $\mathrm{~N}-\mathrm{R}$ & $\mathrm{N}-\mathrm{R}$ & $\overline{2.27}$ & $\overline{2.33}$ & 2.63 & & \\
2.5 & $\overline{1.36}$ & 1.49 & 1.85 & 1.87 & 2.15 & & \\
3.0 & 1.35 & 1.49 & 1.57 & 1.42 & 2.03 & & \\
3.3 & & 0.95 & 1.14 & 1.23 & & & \\
5.0 & & & & & & &
\end{tabular}

Note: N-R, no rocking.
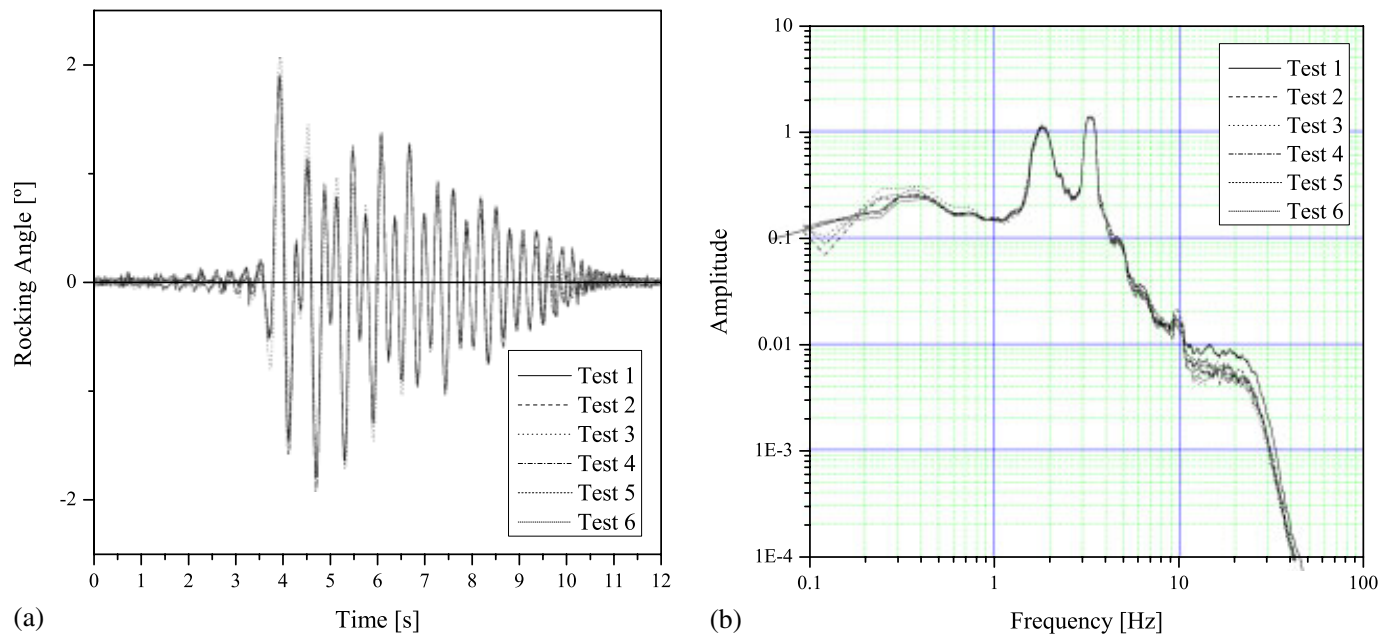

Figure 6. Typical repeatability test carried out with Hanning sine with frequency of $3.3 \mathrm{~Hz}$ and amplitude of $7 \mathrm{~mm}$ (specimen 1): (a) rocking angle and (b) Fourier spectra.

One is related to the load frequency $(3.3 \mathrm{~Hz})$, while the other $(1.9 \mathrm{~Hz})$ is associated with the transient response.

The CCRR and DE models are able to fully reproduce the harmonic motion tests. Figure 7 shows the typical response of RB under constant sine base motion. For clarity of the figure, a gap is made in the time axis between 4 and $19 \mathrm{~s}$ that corresponds to the first part of the stationary state. The responses of both models are almost the same as in the experimental tests even during the last cycles.

\subsection{Random motion}

Specimen 1 was damaged after the set of tests described above. Therefore, only specimens 2-4 were subjected to random motion in order to study the dynamic behavior under earthquake motions. 


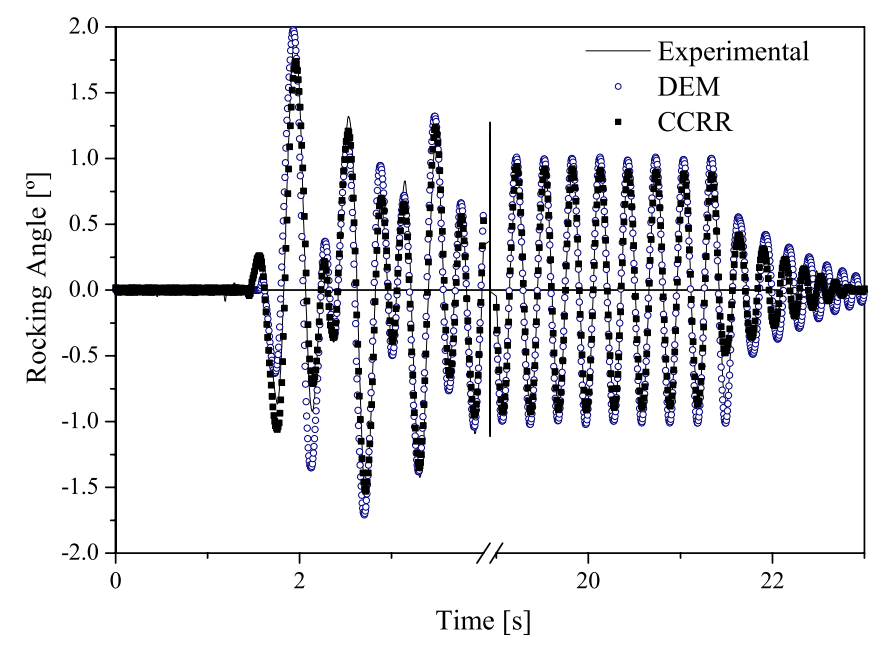

Figure 7. Typical response under harmonic motion (specimen 2, constant sine with frequency of $5 \mathrm{~Hz}$ and $5 \mathrm{~mm}$ of amplitude).

A repeatability test was carried out with the generated random motion, under increasing load factors that multiply the original accelerogram. Each test was repeated at least twice. Typical responses are shown in Figure 8 in terms of rocking angle curves for specimen 2 loaded with earthquake 20 and load factors of 1.1 and 1.3. With a load factor of 1.1, the specimen has a maximum rocking angle of $6.8^{\circ}$ for one test while with the other test the specimen overturns. It is worth noting that during the first $6 \mathrm{~s}$, the behavior of the RB is similar for the two tests. Two tests were carried out with the load factor of 1.3. In this case, the RB did not overturn for any test and both the behaviors are similar during the first few seconds. However, at the end of the load the behaviors became rather different, especially during the free rocking motion. This is mainly due to the small changes in the initial position that always exist in the rocking motion and that cause a change in boundary conditions.

In the same figure, the response of specimen 2 is compared with both models. In the first test (Figure 8(a)), the response of both the models is in good agreement with one experiment but not with the other due to the lack of repeatability. In the test with load factor of 1.3 (Figure 8(b)), the numerical models collapse while the two experimental tests remain stable. However, the response of the models matches well with the experimental tests during the first cycles. This fact suggests that the initial conditions for the models and the experimental test are, in fact, equal. However, during the RM regime these conditions change, allowing the real blocks to survive the collapse.

Figure 9 shows a typical result of random motion with specimen 3. The numerical models are again in good agreement with the experimental test, and they are also successful in predicting the collapse of the specimen.

From the experimental results, it can be seen that the dynamic critical angle is greater than the static critical angle (parameter $\alpha$ ). Here, the dynamic critical angle is defined as the angle at which the stone overturns due to dynamic forces. Table $\mathrm{V}$ shows the average critical angle obtained from the random tests. This should be expected due to the beneficial effect of the inertial forces. Therefore, the static critical angle is a conservative value for the design process. 

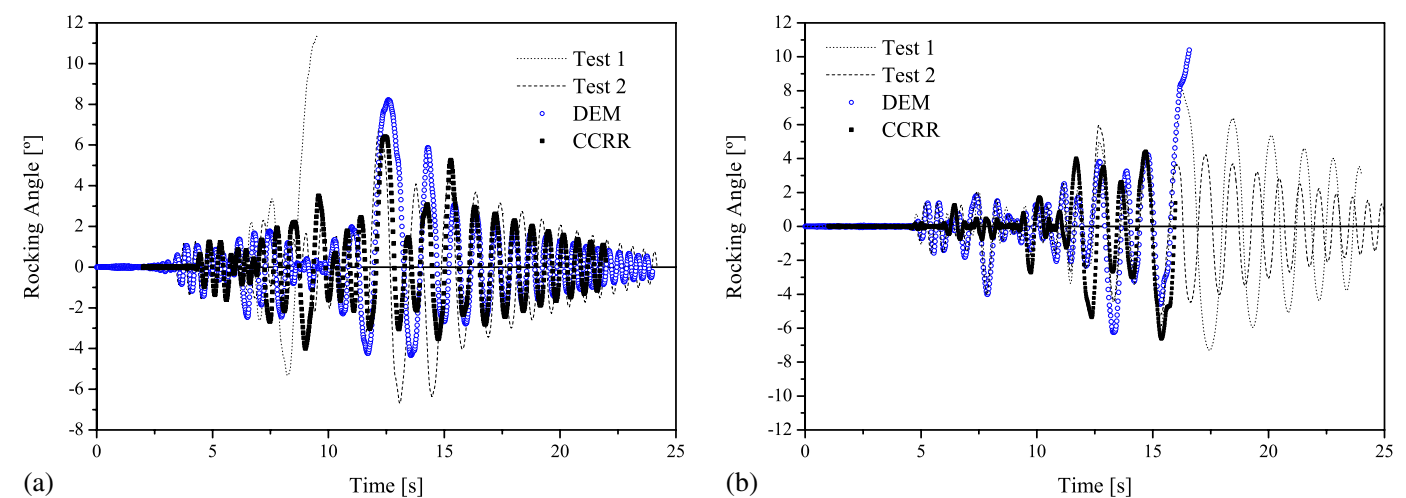

Figure 8. Typical results of random motion (specimen 2): (a) earthquake record 20 and load factor of 1.1 and (b) earthquake record 20 and load factor of 1.3.

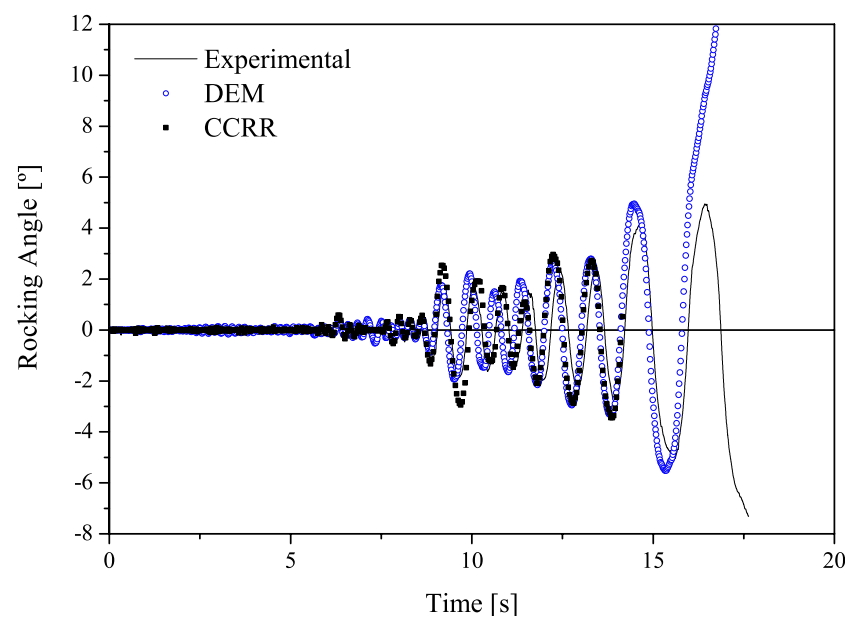

Figure 9. Typical result of random motion (specimen 3, earthquake record 18 and load factor of 0.5 ).

Table V. Critical angle.

\begin{tabular}{lccc}
\hline & \multicolumn{3}{c}{ Critical angle (deg.) } \\
\cline { 2 - 4 } Specimen & Static & Dynamic & Difference (\%) \\
\hline 2 & 9.6 & 11.2 & 16 \\
3 & 6.8 & 6.9 & 2 \\
4 & 18.0 & 20.8 & 15 \\
\hline
\end{tabular}




\section{CONCLUSIONS}

This paper presents a comprehensive experimental program carried out on four blue granite stones under dynamic loading. Two different analytical tools were successfully calibrated and validated with the experimental results. The CCRR and the DEM are useful tools in the study of the rocking motion.

Free rocking motion tests allowed us to obtain the parameters that define rocking $\alpha, \mu$ and $p$. The experimental values of $\alpha$ are lower than the theoretical, while the restitution coefficient $\mu$ is larger. Parameter $p$ does not give a defined pattern. Specimens with low and moderate slenderness (down to 6) present less variation in their parameters, since these stones are nearest to the hypotheses assumed by the classical theory. It is worth noting that the sliding motion was not recorded in any test.

Repeatability of the tests with harmonic ground motion exists. However, it does not exist with random motion, where very large variations are found in the response. This is due to the combination of high frequencies and the small changes in the boundary conditions. The amplitude of rocking depends on the frequency and amplitude of load. The rocking motion is greater for low frequencies.

Both models are extremely sensitive to the classical parameters and small variations in their values produce large differences in the response. This is particularly evident for the parameter $\alpha$, which is related to the geometry of the rocking block. Therefore, fitting parameters were used in order to obtain a good agreement between numerical models and experimental results.

A new methodology has been proposed for finding the parameters of the DE model by using the parameters of the classical theory. This methodology allows us to obtain the parameters used by the DE model in a simple manner. The numerical simulations show that the proposed parameters allow to reproduce the experimental test with good accuracy. An empirical formula to obtain the equivalent viscous damping necessary for defining the DE model has also been proposed. This damping is proportional to the geometry of the block, the stiffness of the joint and the coefficient of restitution $\mu$.

Despite the limitations and difficulties of reproducing the initial and boundary conditions, a good agreement has been found between numerical and experimental responses for both free and forced regimens. For random vibrations, a step forward to stochastic analysis seems to be required, as indicated by the lack of repeatability of the experimental results.

\section{ACKNOWLEDGEMENTS}

The experimental tests carried out in this work were part of the Project ECOLEADER Group 4. F. Peña and F. Prieto acknowledge funding from the FCT grant contracts SFRH/BPD/17449/2004 and SFRH/BD/9014/2002, respectively.

\section{REFERENCES}

1. Augusti G, Sinopoli A. Modelling the dynamics of large block structures. Meccanica 1992; 27:195-211. DOI: 10.1007/BF00430045.

2. Boroschek R, Iruretagoyena A. Controlled overturning of unanchored rigid bodies. Earthquake Engineering and Structural Dynamics 2006; 35:695-711. DOI: 10.1002/eqe.554.

3. Hogen SJ. The many steady states responses of a rigid block under harmonic forcing. Earthquake Engineering and Structural Dynamics 1990; 19:1057-1071. 
4. Housner G. The behavior of inverted pendulum structures during earthquakes. Bulletin of the Seismological Society of America 1963; 53(2):403-417.

5. Ishiyama Y. Motions of rigid bodies and criteria for overturning by earthquake excitations. Earthquake Engineering and Structural Dynamics 1982; 10:635-690.

6. Taniguchi T. Non-linear response analyses of rectangular rigid bodies subjected to horizontal and vertical ground motion. Earthquake Engineering and Structural Dynamics 2002; 31:1481-1500. DOI: 10.1002/eqe.170.

7. Taniguchi T, Miwa T. A simple procedure to approximate slip displacement of freestanding rigid body subjected to earthquake motions. Earthquake Engineering and Structural Dynamics 2007; 36:481-501. DOI: 10.1002/eqe.639.

8. Tso WK, Wong CM. Steady state rocking response of rigid blocks to earthquake. Part 1: analysis. Part 2: experiment. Earthquake Engineering and Structural Dynamics 1989; 18:89-120.

9. Zhang J, Makris N. Rocking response of free-standing blocks under cycloidal pulses. Journal of Engineering Mechanics 2001; 127(5):473-483.

10. Prieto F, Lourenço PB. On the rocking behavior of rigid objects. Meccanica 2005; 40:121-133. DOI: 10.1007/s11012-004-5875-z.

11. Prieto F, Lourenço PB, Oliveira CS. Impulsive Dirac-delta forces in the rocking motion. Earthquake Engineering and Structural Dynamics 2004; 33:839-857. DOI: 10.1002/eqe.381.

12. Azevedo J, Sincrain G, Lemos JV. Seismic behavior of blocky masonry structures. Earthquake Spectra 2000; 16(2):337-365.

13. Hori N, Inoue N, Purushotam D, Nishida T, Kobayashi J. Experimental and analytical studies on earthquake resisting behaviour of confined concrete block masonry structures. Earthquake Engineering and Structural Dynamics 2006; 35:1699-1719. DOI: 10.1002/eqe.604.

14. NTUA. Monuments under seismic action. A numerical and experimental approach. Report No. NTUA/LEE-97/01, Laboratory for Earthquake Engineering, Faculty of Civil Engineering, National Technical University of Athens, 1997.

15. Papantonopoulos C, Psycharis N, Papastamatiou DY, Lemos JV, Mouzakis HP. Numerical prediction of the earthquake response of classical columns using the distinct element method. Earthquake Engineering and Structural Dynamics 2002; 31:1699-1717. DOI: 10.1002/eqe.185.

16. Psycharis N, Lemos JV, Papastamatiou DY, Zambas C, Papantonopolous C. Numerical study of the seismic behaviour of a part of the Parthenon Pronaos. Earthquake Engineering and Structural Dynamics 2003; 32: 2063-2084. DOI: 10.1002/eqe.315.

17. Winkler T, Meguro K, Yamazaki F. Response of rigid body assemblies to dynamic excitation. Earthquake Engineering and Structural Dynamics 1995; 24:1389-1408.

18. Itasca Consulting Group, Inc. UDEC-Universal Distinct Element Code, Version 3.1-User's Guide, CD-ROM, U.S.A., 2000.

19. Sincrain G, Lemos JV. A discrete element program based on a rigid block formulation. Report 40/98-NDE/NEE, Laboratório Nacional de Engenharia Civil, Lisboa, 1998.

20. Yim C, Chopra A, Penzien J. Rocking response of rigid blocks to earthquake. Report UBC/EERC-80/02, University of California, 1980.

21. Eurocode 8. Design provisions for earthquake resistance of structures. Part 1-1. EN 1998-1, CEN, Brussels, 2004.

22. Lourenço PB, Ramos F. Characterization of cyclic behavior of dry masonry joints. Journal of Structural Engineering (ASCE) 2004; 130(5):779-786. 